\title{
Ictioplancton en el estuario del río Pánuco, Tamaulipas-Veracruz
}

\section{Ichthyoplankton in the Pánuco river estuary, Tamaulipas-Veracruz}

\section{Marina Sánchez-Ramírez ${ }^{1 *}$ (D), Ariana Lizbet Berrios- Zamudio ${ }^{1}$ D \\ Alberto Ocaña-Luna}

Laboratorio de Ecología, Departamento de Zoología, Escuela Nacional de Ciencias Biológicas, Instituto Politécnico Nacional, Unidad Profesional Lázaro Cárdenas. Prolongación de Carpio y Plan de Ayala s/n, Col. Santo Tomas, CP. 11340. Alcaldía Miguel Hidalgo, Ciudad de México. México.

*Autor de correspondencia: masanchezra@ipn.mx

Nota científica

Recibida: 10 de octubre 2020

Aceptada: 12 de abril 2021

Como citar: Sánchez-Ramírez $\mathrm{M}$, Berrios-Zamudio

$\mathrm{AL}$,

Ocaña-Luna A (2021) Ictioplancton en el estuario del río Pánuco, Tamaulipas-Veracruz. Ecosistemas y Recursos Agropecuarios 8(1): e2735. DOI: 10.19136/era.a8n1.2735
RESUMEN. Se determinó la variación en la estructura del ensamblaje de larvas de peces a lo largo de un gradiente salino en el estuario del río Pánuco, para lo cual se realizaron muestreos en: enero, marzo, mayo, julio, septiembre y noviembre en 2017 en nueve sitios, con una red estándar con abertura de malla de $500 \mu \mathrm{m}$. Se recolectaron 1165 huevos de peces y 526 larvas de peces de 18 especies, tres de ellas de agua dulce. La mayor riqueza de especies se observó en el estuario medio y laguna de Pueblo Viejo. Los valores mayores de diversidad y equidad se presentaron en noviembre (2.7 y 0.9 , respectivamente). La dominancia fue mayor en julio (0.4) debido a la abundancia de Anchoa mitchilli. El ensamblaje ictioplanctónico se caracterizó por un mayor número de especies dominantes marinas y estuarinas, un bajo número de dulceacuícolas y disminución de la riqueza río arriba.

Palabras clave: Huevos de peces, inmigración, larvas de peces, lagunas costeras, zooplancton.

ABSTRACT. To determine the structure of the ichthyoplankton assemblage along a saline gradient in the Pánuco river estuary, sampling was performed in: January, March, May, July, September, and November in 2017 at nine sites, with a standard network with a mesh opening of $500 \mu \mathrm{m}$. 1 165 eggs and 526 fish larvae were collected from 18 species, three of them from freshwater. The highest specific richness was observed in the middle estuary and lagoon of Pueblo Viejo. The highest values of diversity and evenness was found in November (2.7 and 0.9, respectively). The dominance was greatest in July (0.4) due to the abundance of Anchoa mitchilli. The ichthyoplankton assemblage was characterized by a greater number of dominant marine and estuarine species, a low number of freshwater species and a decrease in species richness upstream.

Key words: Fish eggs, immigration, fish larvae, coastal lagoons, zooplankton. 


\section{INTRODUCCIÓN}

La heterogeneidad ambiental de los estuarios propicia la formación de diferentes hábitats en los que un gran número de especies pueden establecerse (Hoffmeyer 2004). La cuenca del río Pánuco es resultado de la unión de los ríos Moctezuma y Tamuín, se extiende $185 \mathrm{~km}$ hasta Tampico, Tamaulipas donde desemboca en el Golfo de México (Hudson 2000). Este estuario la mayor parte del tiempo se encuentra altamente estratificado con una cuña salina en su parte final, considerado como micromareal por su intervalo medio de marea bajo de 0.40 a $0.60 \mathrm{~m}$ y alta descarga del río (Arcos-Espinosa et al. 2008), que puede alcanzar 16020 millones de $\mathrm{m}^{3}$ anuales (DOF 1998) y descarga media de $426.31 \mathrm{~m}^{3} \mathrm{~s}^{-1}$ (GonzálezRamírez y Parés-Sierra 2019).

Por otro lado, la ictiofauna dulceacuícola ha sido estudiada en varias regiones de la cuenca del Río Pánuco (Soto-Galera et al. 2011, Gutiérrez-Yurita et al. 2013). En los ambientes salobres asociados al estuario del río Pánuco se ha registrado la ictiofauna adulta en la laguna del Chairel (Darnell 1962) y en la laguna de Pueblo Viejo (Castillo-Rivera y ZárateHernández 2001). Para los estudios de ictioplancton en estuarios, sólo existen dos en el estado de Tabasco, el del río Grijalva (Arévalo-Frías y MendozaCarranza 2015) y el del río San Pedro y San Pablo (Arévalo-Frías y Mendoza-Carranza 2012). Por lo que al momento no se tienen investigaciones sobre aspectos de la ictiofauna del estuario del río Pánuco; sitio de una gran complejidad hidrológica y que seguramente alberga una gran diversidad. Por lo que el objetivo del presente estudio fue determinar la variación en la estructura del ensamblaje de larvas de peces a lo largo de un gradiente salino en este estuario, durante un ciclo anual.

\section{MATERIALES Y MÉTODOS}

\section{Área de estudio}

El estuario del río Pánuco se localiza en los límites de los estados de Tamaulipas y Veracruz; el clima es cálido subhúmedo $A_{1}(\mathrm{e})$, presenta una época de secas corta durante el verano y una marcada en invierno (García 1998). Al norte se comunica con la laguna del Chairel, la cual se ubica a 28.5 $\mathrm{km}$ del golfo de México ( $22^{\circ} 10^{\prime} \mathrm{LN}$ y $97^{\circ} 51^{\prime} \mathrm{LO}$ ), cuenta con una superficie de $12.3 \mathrm{~km}^{2}$ (Deegan et al. 1986); mientras que al sur con la laguna de Pueblo Viejo a $9.73 \mathrm{~km}$ del golfo de México $\left(22^{\circ} 05^{\prime}-22^{\circ} 13^{\prime}\right.$ LN y $97^{\circ} 50^{\prime}-98^{\circ} 00^{\prime}$ LO), con una superficie de 89 $\mathrm{km}^{2}$ (Castillo-Rivera y Kobelkowsky 1993) y con la laguna del Carpintero $\left(22^{\circ} 15^{\prime}-22^{\circ} 14^{\prime} \mathrm{LN}\right.$ y $97^{\circ} 52^{\prime}-97^{\circ}$ 52' LO), en el centro de la ciudad de Tampico, con 0.9 $\mathrm{km}^{2}$ de superficie (Crisóstomo-Vázquez et al. 2016).

Para la recolecta del ictioplancton se realizaron seis muestreos bimestrales (enero, marzo, mayo, julio, septiembre y noviembre) durante el 2017 en nueve sitios, localizados en cuatro regiones: estuario alto (estaciones 1 y 2), laguna del Chairel (estaciones 6 y 7), estuario medio (estaciones 3,4 y 5 ) y laguna de Pueblo Viejo (estaciones 8 y 9) (Figura 1), mediante arrastres en la capa superficial de la columna de agua utilizando una red estándar con abertura de malla de $500 \mu \mathrm{m}$, longitud de $150 \mathrm{~cm}$, diámetro de la boca de $50 \mathrm{~cm}$ y flujómetro General Oceanics adaptado a la misma, para calcular el volumen de agua filtrado. De manera simultánea se tomaron datos de temperatura superficial en ${ }^{\circ} \mathrm{C}$ con un termómetro de mercurio y la salinidad en ups con un refractómetro.

En cada muestra se separó el total de huevos y larvas de peces, para determinar el taxón más bajo posible. Se estimó la abundancia, la cual se expresó en términos de la densidad como número de huevos o larvas por cada $100 \mathrm{~m}^{-3}$. La estructura del ensamblaje se analizó considerando la riqueza, la cual se estimó como número de especies; para determinar las especies dominantes se utilizó el índice de valor de importancia (IVI), modificado de Cottam y Curtis (1956) la diversidad mensual se calculó a través del índice de Shannon-Wiener $\left(H^{\prime}\right)$ (Shannon y Weaver 1964) y se expresó como bits ind ${ }^{-1}$, se realizó una prueba de $t$ (Hutcheson 1970) para establecer la diferencias temporales de este parámetro; la dominancia, se obtuvo mediante el índice de Simpson (Simpson 1949); para calcular la equidad se empleó el índice de Pielou (Pielou 1966). Para luego 


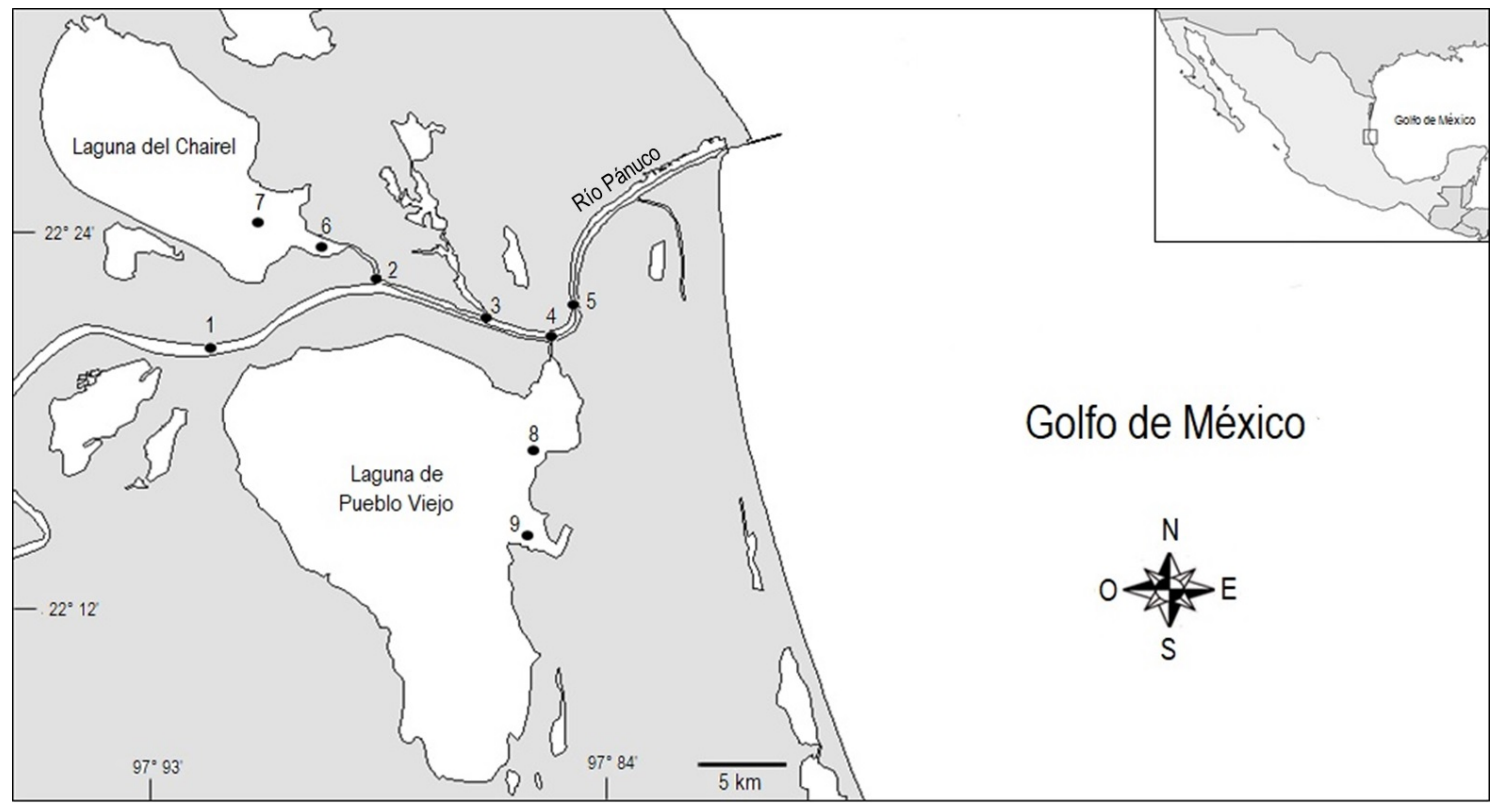

Figura 1. Área de estudio y ubicación de las estaciones de muestreo en el estuario del río Pánuco, Tamaulipas- Veracruz.

determinar la conformación de grupos en un dendrograma definido por el índice de disimilitud de Bray-Curtis (Bray y Curtis 1957), por grupos pareados, a partir de los datos de densidad transformados $(\ln [x+1])$, para lo cual se utilizó el programa Past versión 3.21 .

\section{RESULTADOS Y DISCUSIÓN}

Se recolectaron 1165 huevos de peces y 526 larvas de peces que pertenece a 13 familias, 16 géneros y 18 especies, tres de ellas de agua dulce. La mayor riqueza de especies se observó en el estuario medio y laguna de Pueblo Viejo (18 especies), con disminución río arriba en la región alta del estuario y laguna del Chairel (12 especies). Las especies Anchoa mitchilli, Dormitator maculatus, Ctenogobius boleosoma y Lagodon rhomboides, estuvieron presentes en todo el sistema; Catostomidae sp. 1, Ulaema lefroyi y Conodon nobilis sólo en el estuario medio y Cynoscion arenarius sólo en la laguna de Pueblo Viejo (Tabla 1). La mayor riqueza de especies se observó de julio a noviembre (15), mientras que la menor de enero a mayo (12).
El registro de 18 especies en el estuario del río Pánuco es similar con lo observado en el estuario de los ríos Grijalva, San Pedro-San Pablo en el estado de Tabasco, donde se observaron 14 y 18 especies, respectivamente (Árevalo-Frías y Mendoza-Carranza 2012), así como con el estuario del río de la Plata en Argentina-Uruguay donde se recolectaron 18 especies (Berasategui et al. 2004), estos ríos presentan un caudal elevado y régimen micromareal (LópezLaborde y Nagy 1999, Arcos-Espinosa et al. 2008, Arcos-Espinosa et al. 2010). Esto difiere de lo que ocurre en otro tipo de estuarios, donde el caudal es bajo y la marea presenta un régimen mesomareal, el número de especies aumenta considerablemente como en el caso de los estuarios Guadiana y Lima en Portugal con la presencia de 34 y 50 especies de larvas de peces, respectivamente (Faria et al. 2006, Ramos et al. 2006).

Se recolectaron huevos de $A$. mitchilli, con la mayor abundancia promedio anual en el sistema (98.13\%) en sitios con temperatura de 23.5 a $31.0{ }^{\circ} \mathrm{C}$ y salinidad de 1.0 a 23.0 ups (Tabla 2), con mayor concentración en las lagunas el Chairel y Pueblo Viejo, así como en la zona media del estuario, por lo que el desove de $A$. mitchilli es comparable a lo 
Tabla 1. Presencia $(X)$ de especies de larvas de peces por región. Estuario del río Pánuco, Tamaulipas-Veracruz.

\begin{tabular}{|c|c|c|c|c|}
\hline Especie/Región & EA & LC & EM & LPV \\
\hline Anchoa hepsetus & & & $\mathrm{X}$ & $\mathrm{X}$ \\
\hline Anchoa mitchilli & $\mathrm{x}$ & $\mathrm{x}$ & $\mathrm{x}$ & $\mathrm{x}$ \\
\hline Cyprinidae sp. & $\mathrm{x}$ & & $\mathrm{x}$ & \\
\hline Catostomidae sp. 1 & & & $x$ & \\
\hline Catostomidae sp. 2 & $\mathrm{X}$ & & $\mathrm{x}$ & \\
\hline Dormitator maculatus & $x$ & $\mathrm{x}$ & $x$ & $\mathrm{x}$ \\
\hline Ctenogobius boleosoma & $\mathrm{x}$ & $\mathrm{x}$ & $\mathrm{x}$ & $\mathrm{x}$ \\
\hline Gobionellus oceanicus & & $\mathrm{x}$ & $\mathrm{x}$ & $\mathrm{x}$ \\
\hline Gobiosoma bosc & & $\mathrm{x}$ & $\mathrm{x}$ & $\mathrm{x}$ \\
\hline Gobiosoma robustum & & $\mathrm{x}$ & & $\mathrm{x}$ \\
\hline Lupinoblennius nicholsi & $\mathrm{X}$ & & $\mathrm{x}$ & $\mathrm{x}$ \\
\hline Membras martinica & & & $x$ & $\mathrm{x}$ \\
\hline Achirus lineatus & $\mathrm{x}$ & $\mathrm{x}$ & & $\mathrm{x}$ \\
\hline Microphis lineatus & $\mathrm{x}$ & & $\mathrm{x}$ & $\mathrm{x}$ \\
\hline Ulaema lefroyi & & & $\mathrm{x}$ & \\
\hline Conodon nobilis & & & $\mathrm{x}$ & \\
\hline Cynoscion arenarius & & & & $\mathrm{X}$ \\
\hline Lagodon rhomboides & $\mathrm{x}$ & $\mathrm{x}$ & $\mathrm{x}$ & $x$ \\
\hline Intervalo de salinidad (ups) & $0.0-16.0$ & $0.0-14.0$ & $0.0-12.0$ & $1.0-24.0$ \\
\hline Salinidad promedio (ups) & 3.2 & 5.6 & 4.8 & 14.3 \\
\hline Intervalo de temperatura $\left({ }^{\circ} \mathrm{C}\right)$ & $23.0-29.5$ & $23.5-30.0$ & 23.5-31.0 & $24.9-31.0$ \\
\hline Temperatura promedio $\left({ }^{\circ} \mathrm{C}\right)$ & 26.9 & 27.3 & 27.2 & 28.0 \\
\hline
\end{tabular}

que ocurre en la Laguna de Pueblo Viejo en el estado de Veracruz (Sánchez-Ramírez y Ocaña-Luna 2002). La mayor densidad de larvas de peces se presentó en los meses de marzo y septiembre (41.7 y 52.9 larvas $100 \mathrm{~m}^{-3}$, respectivamente). Anchoa mitchilli fue la especie dominante $(\mathrm{IVI}=91.4)$ (Tabla 2$)$, la cual se recolectó todo el año con una abundancia promedio anual de $37.7 \%$ en sitios con temperaturas de 22.5 $31.0^{\circ} \mathrm{C}$ y salinidades de 0.0 a 24.0 ups; por otro lado, Cyprinidae sp. y Catostomidae sp. 2, especies de agua dulce, que se localizaron en sitios con intervalos reducidos de temperatura $\left(26.0-28.0^{\circ} \mathrm{C}\right)$ y 0.0 ups.

La diversidad de larvas de peces en el estuario del río Pánuco presentó el mayor valor en noviembre de 2.7 bits ind ${ }^{-1}(p<0.0001)$; se ha observado que hacia el norte en la laguna Madre en Tamaulipas la diversidad se mantiene entre 1.02-2.23 (SánchezRamírez y Ocaña-Luna 2015), mientras que hacia el sur del río Pánuco en las lagunas Tamiahua y Tampamachoco en Veracruz, puede alcanzar valores de 2.13 a 3.30 y de 1.8 a 3.10, respectivamente (OcañaLuna y Sánchez-Ramírez 2003, 2016); por otro lado a nivel del Ecuador entre latitudes de $2^{\circ}$ y $3^{\circ}$ se observa una diversidad menor de dos, en el estuario del río
Bacanga en el noreste de Brasil se registraron valores entre 0.08 y 0.99 (Barbosa-Soares et al. 2014), en el estuario interno del Río Guayas, Ecuador fue de 0.81 a 1.5 (Calderón-Peralta et al. 2020), lo que coincide con De Lafontaine (1990) quién señala que los estuarios tienen una diversidad significativamente menor a dos, mientras que en la zona costera marina y bahías mayor a dos; pero difiere de lo que ocurre en el estuario del Río Pánuco, sitio que se encuentra inmerso en la Región Biogeográfica Caribeña y con influencia de la Región Neártica, por lo que esta ubicación geográfica puede influir en el aumento de la diversidad. Por otra parte, la dominancia fue mayor en julio (0.4), por la abundancia de A. mitchilli, mientras que la equidad fue menor (Tabla 2).

El ensamblaje de larvas de peces, con base a la abundancia y frecuencia de los taxa, quedó conformado por cuatro grupos (Figura 2): Grupo I) especies más abundantes o exclusivas del mes de noviembre; Grupo II) integrado por las especies con abundancia relativamente baja y taxa de agua dulce, presentes sólo en septiembre o con su mayor abundancia en este mes; Grupo III) especies dominantes y Grupo IV) especies exclusivas de marzo. Por último 
Tabla 2. Abundancia promedio mensual (Núm. de individuos $/ 100 \mathrm{~m}^{3}$ ) y promedio anual del ictioplancton (\%), Índice de Valor de Importancia (IVI) y parámetros ecológicos. Estuario del río Pánuco, Tamaulipas-Veracruz.

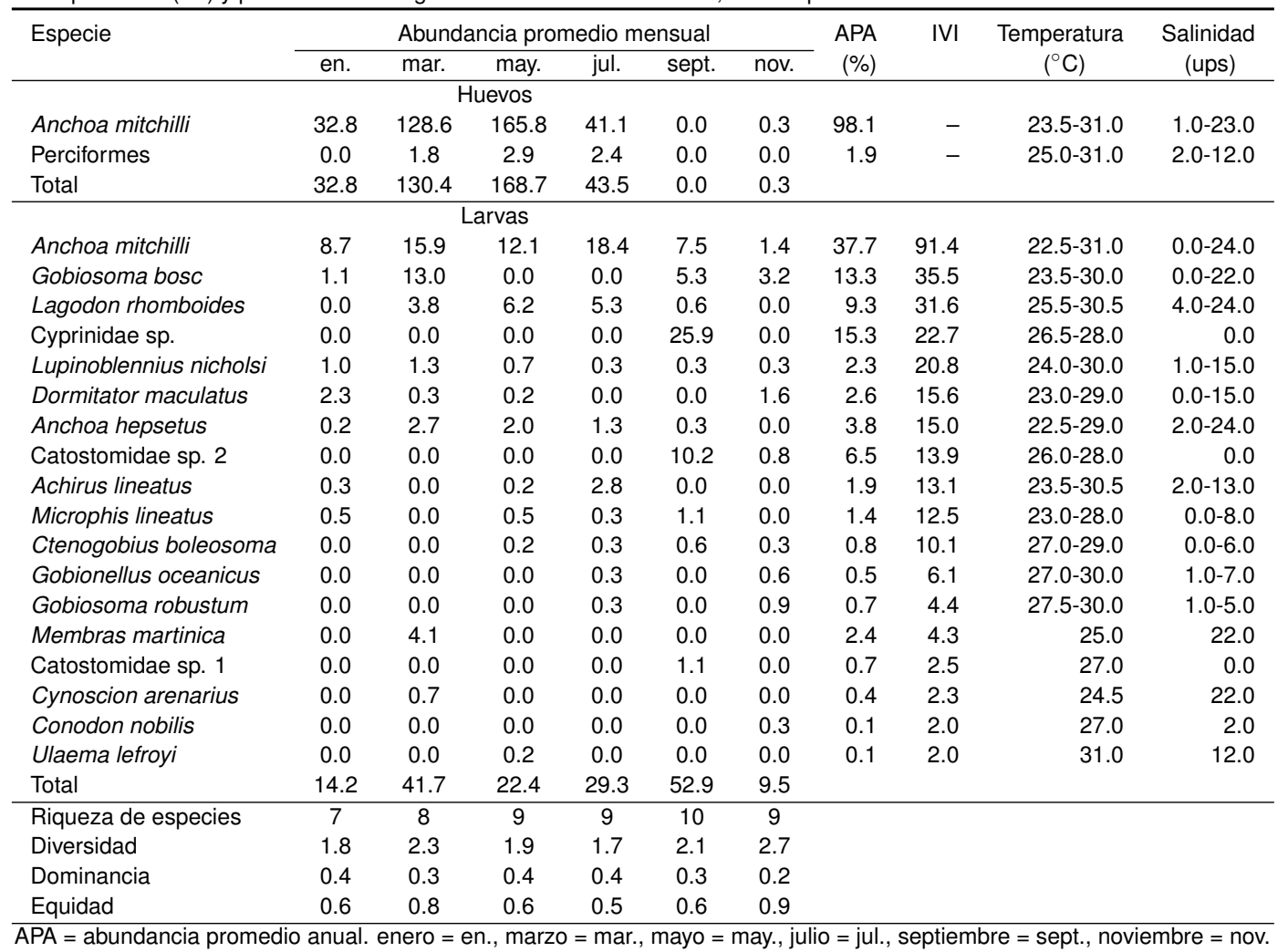

$U$. lefroyi fue excluida de los grupos por ser única en el mes de mayo y con abundancia mínima. Dentro del grupo dominante destaca A. mitchilli, especie residente permanente lagunar al igual que en otras lagunas costeras de Tamaulipas y norte del estado de Veracruz como: laguna Madre (Sánchez-Ramírez y Ocaña-Luna 2015), laguna de Tamiahua (OcañaLuna y Sánchez-Ramírez 2016) y laguna de Tampamachoco (Ocaña-Luna y Sánchez-Ramírez 2003). En el sur de Tamaulipas los adultos penetran al Sistema Guayalejo-Tamesí, tributario del río Pánuco, junto con otras especies como A. hepsetus, C. boleosoma y G. bosc (García de Léon et al. 2005).

En lo que respecta al componente dulceacuícola, sólo se registraron tres especies, dos de la familia Catostomidae y una de la familia Cyprinidae; en estuarios con mayor caudal suele presentarse un mayor número de especies de agua dulce, a diferencia de lo que ocurre cuando la intrusión salina es mayor, hecho que ha sido observado en el estuario
Lima por Ramos et al. (2006). Las dos especies de la familia Catostomidae no pudieron ser determinadas, sin embargo pueden corresponder al género lctiobus que es el único registrado en las diferentes cuencas que confluyen hacia el Río Pánuco, con la especie Ictiobus labiosus en la cuenca del Río Moctezuma (subcuenca tramo medio) (Gutiérrez-Yurita et al. 2013); Ictiobus bubalus e I. labiosus se han catalogado como especies primarias de agua dulce y registradas en el sistema Guayalejo-Tamesí (García de León et al. 2005), así como en los ríos Tamuín, Moctezuma y Pánuco (Soto-Galera et al. 2011). Las especies de las familias Catostomidae y Cyprinidae, se distribuyeron en las regiones alta y media del estuario del río Pánuco (Tabla 1), principalmente durante la época de lluvias (septiembre-noviembre), esta agrupación de larvas de peces con afinidad dulceacuícola es común en la región río arriba. Otra especie de este componente dulceacuícola fue Microphis lineatus, señalada como una especie típica de agua dulce 


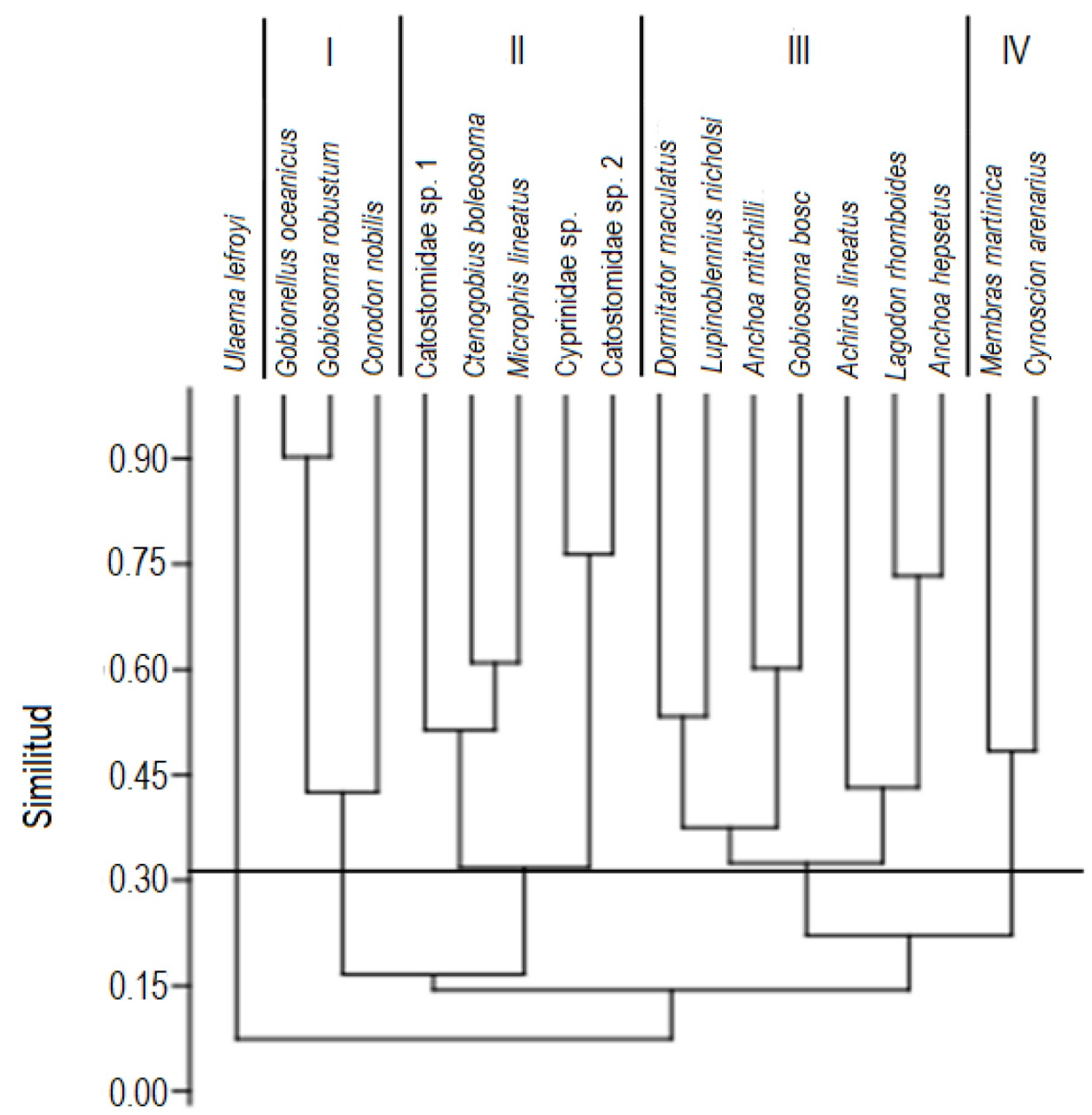

Figura 2. Dendrograma de similitud de especies a partir de la abundancia de larvas de peces (larvas/100 $\mathrm{m}^{3}$ ) definido por el índice de disimilitud de Bray-Curtis, por grupos pareados. Estuario del río Pánuco, TamaulipasVeracruz (2017).

por Gilmore y Gilbert (1992); sus larvas se registraron en las tres épocas climáticas presentes en la zona (nortes, secas y lluvias), con densidad mayor en el mes de septiembre, hecho que coincide con lo observado en la laguna de Tamiahua (Ocaña-Luna y Sánchez-Ramírez 2016), es importante mencionar que $M$. lineatus es una de las pocas especies cuyos machos liberan larvas planctónicas con una talla de $6.0 \mathrm{~mm}$ (Frías-Torres 2004).
El ensamblaje de larvas en el estuario del río Pánuco tuvo mayor número de especies marinas dominantes y estuarinas permanentes a lo largo del año y un bajo número de especies dulceacuícolas durante el periodo de lluvias, estos hechos están influenciados principalmente por un gran flujo de agua dulce y bajo nivel mareal, transporte pasivo mareal de las especies en la época de estiaje (enero a julio), por lo que se puede considerar como una región de tránsito hacia las zonas de crianza: lagunas del Chairel, del Carpintero y de Pueblo Viejo. 


\section{LITERATURA CITADA}

Arcos-Espinosa G, Jiménez Hernández SB, Padilla-Hernández R (2010) Escurrimiento medio anual y disponibilidad de agua en la vertiente del Golfo de México. En: Rivera-Arriaga E, Azuz-Adeath I, Alpuche Gual L, Villalobos-Zapata GJ (eds). Cambio Climático en México un Enfoque Costero-Marino. Universidad Autónoma de Campeche CETYS-Universidad, Gobierno del Estado de Campeche. Campeche. pp: 6172.

Arcos-Espinosa G, Medina-Santamaría R, Méndez-Incera FJ, Jiménez-Hernández SB (2008) Estudio de la cuña salina: río Pánuco, México. Ingeniería Hidráulica en México 23: 77-88.

Arévalo-Frías W, Mendoza-Carranza M (2012) Larvas y juveniles de peces en ambientes estuarinos de la Reserva de la Biosfera Pantanos de Centla y su zona costera adyacente. En: Sánchez AJ, Chiappa-Carrara $X$, Brito-Pérez R (eds). Recursos acuáticos costeros del sureste: Tendencias actuales en investigación y estado del arte. Vol. 2. RECORECOS, CONCYTEY, UNACAR, UJAT, ECOSUR, UNAM. México, México. pp: 242-369.

Arévalo-Frías W, Mendoza-Carranza M (2015) Influence of temporal and spatial factors on abundance and richness of fish early stages in shallow tropical estuaries. Environmental Biology of Fishes 98: 891-904.

Barbosa-Soares RD, Jansen-Cutrim MV, Alves-da Silveira PC (2014) Diversidade de larvas de peixes da bacia hidrográfica do río Bacanga (Maranhão, Brazil). Revista de Ciências Ambientais 8: 5-19.

Berasategui AD, Acha U, Fernández-Araoz NC (2004) Spatial patterns of ichthyoplankton assemblages in the Río de la Plata Estuary (Argentina-Uruguay). Estuarine, Coastal and Shelf Science 60: 599-610.

Bray JR, Curtis JT (1957) An ordination of the upland forest communities of Southern Wisconsin. Ecological Monographs 27: 325-349.

Calderón-Peralta G, Ayora-Macías G, Solís-Coello P (2020) Temporal space-variation of fish larvae in the Gulf of Guayaquil, Ecuador. Bulletin of Marine and Coastal Research 49: 135-156.

Castillo-Rivera M, Kobelkowsky A (1993) Comportamiento ambiental de la Laguna de Pueblo Viejo, Veracruz, México. Biotam 5: 1-12.

Castillo-Rivera M, Zarate-Hernández R (2001) Patrones espacio-temporales de la abundancia de peces en la Laguna de Pueblo Viejo, Veracruz. Hidrobiológica 11: 75-84.

Cottam G, Curtis JT (1956) The use of distance measures in phytosociological sampling. Ecology 37: 451-460.

Crisóstomo-Vázquez L, Alcocer-Morales C, Lozano-Ramírez C, Rodríguez-Palacio MC (2016) Fitoplancton de la laguna del Carpintero, Tampico, Tamaulipas, México. Interciencia 41: 103-109.

Darnell RM (1962) Fishes of the río Tamesí and related coastal lagoons in east-central Mexico. Publications of the Institute of Marine Science 8: 299-365.

Deegan LA, Day JW, Gosselink JG, Yañez-Arancibia A, Chávez GS, Sánchez-Gil P (1986) Relationships among physical characteristics, vegetation distribution and fisheries yield in Gulf of Mexico estuaries. In: Wolfe DA (ed) Estuarine variability. Academic Press. New York, USA. pp: 83-100.

De Lafontaine Y (1990) Ichthyoplankton communities in the St. Lawrence estuary: Composition and dynamics. In: El-Sabh MI, Silverberg N (eds.) Oceanography ofa large-scale estuarine system. The St. Lawrence. Lecture Notes on Coastal and Estuarine Studies. Springer-Verlag, New York. USA. pp: 321-343.

DOF (1998) Estudios técnicos para determinar la disponibilidad de las aguas superficiales de la cuenca del río Pánuco. Diario Oficial de la Federación. México. pp: 7-9. 
Faria A, Morais P, Chícharo MA (2006) Ichthyoplankton dynamics in the Guadiana estuary and adjacent coastal area, South-East Portugal. Estuarine, Coastal and Shelf Science 70: 85-97.

Frías-Torres S (2004) Notes on aquarium brood release and feeding of the opossum pipefish, Microphis brachyurus lineatus. Gulf and Caribbean Research 16: 73-75.

García E (1998) Modificaciones al sistema de clasificación climática de Köpen. Universidad Nacional Autónoma de México. México. 90p.

García de León FJ, Gutiérrez-Tirado D, Hendrickson DA, Espinosa-Pérez H (2005) Fishes of the continental waters of Tamaulipas: Diversity and conservation status. In: Cartron J-LE, Ceballos G, Felger RS (ed) Biodiversity, ecosystems and conservation in Northern Mexico. Oxford University Press. New York, USA. pp: 138-166.

Gilmore RG, Gilbert CR (1992) Coastal habitat description (introduction) and species accounts for Microphis brachyurus lineatus. In: Gilbert CR (ed) Rare and endangered biota of Florida: Fishes. Vol 2. University Presses of Florida. Gainesville, USA. pp: 73-78.

González-Ramírez J, Parés-Sierra A (2019) Streamflow modeling of five major rivers that flow into the Gulf of Mexico using SWAT. Atmósfera 32: 261-272.

Gutiérrez-Yurita PJ, Morales-Ortiz JA, Marín-García L (2013) Diversidad biológica, distribución y estrategias de conservación de la ictiofauna de la cuenca del río Moctezuma, centro de México. Limnetica 32: 215-228.

Hoffmeyer M (2004) Decadal change in zooplankton seasonal succession in the Bahía Blanca estuary, Argentina, following introduction of two zooplankton species. Journal of Plankton Research 26: 181-189.

Hudson PF (2000) Discharge, sediment, and channel characteristics of the Río Pánuco, Mexico. Conference of Latin Americanist Geographers 26: 61-70.

Hutcheson K (1970) A test for comparing diversities based on the Shannon formula. Journal of Theoretical Biology 29: 151-154.

López-Laborde J, Nagy GJ (1999) Hydrography and Sediment Transport Characteristics of the Rio de la Plata: A Review. In: Perillo GME, Piccolo MC, Pino-Quivira M (ed) Estuaries of South America: Their Geomorphology and Dynamics. Springer-Verlag Berlin Heidelberg. Alemania. pp: 133-159.

Ocaña-Luna A, Sánchez-Ramírez M (2003) Diversity of ichthyoplankton in Tampamachoco Lagoon, Veracruz, Mexico. Anales del Instituto de Biología, Serie Zoología 74: 179-193.

Ocaña-Luna A, Sánchez-Ramírez M (2016) Estructura de la comunidad ictioplanctónica en la laguna de Tamiahua, Veracruz, México. Revista Mexicana de Biodiversidad 87: 123-132.

Pielou EC (1966) The measurement of diversity in different types of biological collections. Journal of Theoretical Biology 13: 131-144.

Ramos S, Cowen RK, Ré P, Bordalo AA (2006) Temporal and spatial distributions of larval fish assemblages in the Lima estuary (Portugal). Estuarine, Coastal and Shelf Science 66: 303-314.

Sánchez-Ramírez M, Ocaña-Luna A (2002) Temporal variability in the abundance of the bay anchovy Anchoa mitchilli (Valenciennes, 1848) eggs and spawning biomass in Pueblo Viejo Lagoon, Veracruz, Mexico. Hidrobiológica 12: 157-162.

Sánchez-Ramírez M, Ocaña-Luna A (2015) Estructura y variación estacional de la comunidad ictioplanctónica en una laguna hipersalina del oeste del Golfo de México: Laguna Madre, Tamaulipas. Hidrobiológica 25: 175-186. 
Shannon CE, Weaver W (1964) The mathematical theory of comunication. The University of Illinois Press. Urbana, USA. 125p.

Simpson EH (1949) Measurement of diversity. Nature 163: 688.

Soto-Galera E, Alcántara-Soria L, Paulo-Maya J (2011) Estado actual de la ictiofauna dulceacuícola de la provincia del complejo Tamesí-Pánuco. Instituto Politécnico Nacional. Escuela Nacional de Ciencias Biológicas. Informe final SNIB-CONABIO proyecto No. FM027. México. 15p. 